Möglichkeiten kooperativer Politikstile am Beispiel von Holzmöbeln und Kühlgeräten

\title{
Deutsche IPP quo vadis?
}

\author{
Integrierte Produktpolitik IPP setzt auf einen kooperativen Politikstil im Sinne einer lernenden \\ Politik. Wie aber soll dies in der Praxis umgesetzt werden? Wo und wie kann begonnen wer- \\ den? An zwei Produktbeispielen werden Voraussetzungen für und Anforderungen an IPP auf- \\ gezeigt und praktische Möglichkeiten für akteursorientierte Pilotprojekte skizziert.
}

W

Von Esther Hoffmann und Ulla Simshäuser ie können angesichts globaler Märkte und der internationalen Herausforderungen des Umwelt- und Ressourcenschutzes (Agenda 21) wesentliche ökologische Verbesserungen erzielt werden? Die Idee der Integrierten Produktpolitik setzt darauf, dass sich die am Lebensweg eines Produktes beteiligten Akteure auf gemeinsame, übergeordnete Nachhaltigkeitsziele für das Produkt einigen sowie entsprechende Maßnahmen entwickeln und koordinieren. Das IÖW hat im Rahmen eines Gutachtens für das Bundesumweltministerium (BMU) zur Konkretisierung der Ausgestaltung einer IPP zwei Beispiele - Holzmöbel und Kühlgeräte - untersucht (1). In jeweils elf Intensivgesprächen wurden zentrale Akteure wie Hersteller, Handel, Entsorger, VerbraucherInnen und Staat zu einer IPP in ihrem Produktbereich befragt.

\section{Ausgangssituation der Branchen}

Bei den gewählten Produkten Holzmöbel und Kühlgeräte handelt es sich um langlebige Konsumgüter, für die ein gesättigter Markt mit einem intensiven Preiswettbewerb besteht. Während aber im Bereich Kühlgeräte nur wenige große globale Anbieter vorhanden sind, ist der Holzmöbelmarkt durch viele kleine und mittelständische Anbieter geprägt. Bei Kühlgeräten übt insbesondere die wachsende Importquote von billigen und ökologisch eher ungünstigen Geräten einen Druck auf den Markt aus. Gleichwohl sind die Markenhersteller auf dem deutschen Markt ökologisch aufgeschlossen. Es scheint, als ob Ökologie, insbesondere unter dem Gesichtspunkt der Energieeinsparung, als zusätzliches Verkaufsargument im gesättigten Markt herangezogen wird. Demgegenüber ist das Thema Ökologie auf dem Holzmöbel-Markt noch relativ unbedeutend, ob- wohl eine Reihe von Unternehmen ökologische Herausforderungen angenommen hat und ökologische Maßnahmen offensiv anwendet.

\section{Ökologische Herausforderungen der Produkte...}

Küblgeräte wurden in den letzten Jahren ökologisch stark verbessert. Einschlägig ist der kontinuierlich verringerte Energieverbrauch von Neugeräten und der Verzicht auf das klimaschädigende FCKW als Kühlmittel. Größere ökologische Herausforderungen bei der Rohstoffgewinnung, den Vorprodukten und der Herstellung sind nicht zu nennen. Allerdings müssen einzelne Produktbestandteile wie Lacke, Kabel und Schalter weiter optimiert und energieeffiziente Kühlsysteme weiter entwickelt werden. Ökologische Probleme ergeben sich insbesondere durch die Nutzung. Altgeräte müssten zügig durch energiesparende Neugeräte ersetzt werden. Andererseits ist die Nutzungskaskade bedeutsam, da vielfach Altgeräte nicht entsorgt, sondern als Zweitgeräte weiter genutzt bzw. verkauft und exportiert werden.

Eine große Brisanz hat nach wie vor die Entsorgung von Kühlgeräten. Dies betrifft die Sicherstellung eines angemessenen Umgangs mit Altgeräten in der inländischen Entsorgung, vor allem hinsichtlich der FCKW-Erfassung, und die Exporte der Altgeräte in Länder ohne angemessene Entsorgungsstrukturen.

Ökologische Aspekte wurden im Bereich der Holzmöbel bisher vergleichsweise wenig behandelt. Entlang des Produktlebensweges bestehen verschiedene ökologische Herausforderungen. Bei der Rohstoffgewinnung ist dies zum einen die Art der Forstwirtschaft, die - wenn sie nicht ökologisch nachhaltig ist - zu einem Verlust der Artenvielfalt beitragen kann. In der weiteren Verarbeitung der Holzmöbel sind Einsatzstoffe, etwa
Lacke und Klebstoffe, sowie Emissionen, insbesondere flüchtige organische Verbindungen (VOC) aus der Lackierung, ökologisch relevant. Während der Möbeltransport eine vergleichsweise geringe ökologische Bedeutung hat, hat die Nutzung der Möbel mit Blick auf die teilweise kurze Nutzungsdauer sowie auf mögliche gesundheitsschädliche Emissionen von VOC und Formaldehyd ein größeres Gewicht. Die Entsorgung der Möbel stellt zudem sowohl unter dem Mengenaspekt als auch aufgrund der Zusammensetzung ein nicht unbeträchtliches Abfallproblem dar.

\section{... und die Einschätzung ihrer Bedeutung}

Im Rahmen unserer Recherchen gingen wir auch der Frage nach, ob es bei den verschiedenen Akteuren eine einheitliche Bewertung der wichtigsten ökologischen Herausforderungen ihrer Produktgruppe gibt.

Im Ergebnis zeigt sich bei den Küblgeräten ein weitgehender Konsens der Akteure hinsichtlich der Bewertung ökologisch sensibler Bereiche. Als sehr bedeutsame Probleme werden Fragen des Energieverbrauchs der Geräte sowie der Erfassung und Entsorgung von FCKW-Altgeräten angesprochen. Übereinstimmung zwischen den Akteuren deutet sich auch in Bezug auf die Notwendigkeit einer stärkeren umweltbezogenen Kooperation der Akteure entlang des Produktlebensweges an.

Im Bereich der Holzmöbel divergiert dagegen die Einschätzung zu Umweltproblemen zwischen den verschiedenen Akteuren stark. Die meisten Interviewpartner sehen eine Phase des Lebensweges als besonders relevant an. Diese Phase differiert aber je nach befragtem Akteur und stimmte mit dem jeweiligen Haupthandlungsfeld überein. Fast keiner der befragten Akteure hatte den gesamten Lebensweg im Blick; auch die bisherigen Maßnahmen und Vorschläge konzentrierten sich zumeist auf einen Ausschnitt des Produktlebensweges.

\section{IPP-relevante Aktivitäten}

Um den beschriebenen zentralen ökologischen Herausforderungen zu begegnen, wurden von unterschiedlichen Akteuren Maßnahmen ergriffen, die für eine IPP relevant sind.

Für den Bereich der Kühlgeräte zeigt Tabelle 1, dass zu allen identifizierten ökologisch sensiblen Bereichen von unterschiedlichen Akteuren bereits Maßnahmen durchgefuihrt wurden. Schwer- 


\begin{tabular}{|c|c|c|c|}
\hline & \multicolumn{3}{|c|}{ Maßnahmen in zentralen Problembereichen } \\
\hline & Freisetzung von FCKW & Entsorgung & Hoher Energieverbrauch \\
\hline Staatliche & - FCKW-Halon-Verbots- & - Kreislaufwirtschafts-/ & - Öffentliches \\
\hline \multirow[t]{6}{*}{ Maßnahmen } & Verordnung & Abfallgesetz & Beschaffungswesen \\
\hline & - Entsorgungshandbücher & - EG-Entwurf zur & - EU-Forschungsprojekte \\
\hline & - UBA-Leiffaden: & Elektronikschrottverordnung & - Verbraucherinformation/ \\
\hline & Entsorgung von Kältegeräten & - Neuentwurf der & Warentests \\
\hline & & Elektroaltgeräteverordnung & \\
\hline & & - TA Siedlungsabfall & \\
\hline Maßnahmen & - freiwillige Selbstverpflichtung & - Demontagen & - Freiwillige Vereinbarungen \\
\hline \multirow[t]{4}{*}{ anderer Akteure } & des Branchenverbandes ZVEI & & - Öko-Pass des Handels \\
\hline & - Kampagnen (z.B. Greenpeace/ & & - Energielabel \\
\hline & Foron zur Einführung FCKW-freier & & - Erforschung systemischer \\
\hline & Kühlschränke) & & Kühlkonzepte \\
\hline & & & - Vakuumpanels \\
\hline
\end{tabular}

Quelle: eigene Darstellung punkte bildeten vor allem Maßnahmen zur Senkung des Energieverbrauchs der Geräte und der Ersatz von FCKW.

Auch bei den Holzmöbeln wurden für die vier zentralen Problembereiche bereits von verschiedenen Akteuren Maßnahmen ergriffen (vgl. Tabelle 2). Bisher sind ökologische Verbesserungen und Erfolge vor allem im Bereich der Emissionen festzustellen. Für dieses Problemfeld wurden die meisten Maßnahmen durchgeführt. Charakteristisch hinsichtlich der Voraussetzungen für eine IPP ist, dass in beiden Produktbereichen die Maßnahmen noch unverbunden nebeneinander stehen und ein produktpolitisches Gesamtkonzept bisher nicht vorhanden ist. Darüber hinaus weisen die beiden Produktgruppen unterschiedliche Ausgangsbedingungen für eine IPP auf.

- Für Kühlgeräte ist bereits eine profunde Basis vorhanden, da lebenswegübergreifende Betrachtungen und Kooperationen zwischen verschiedenen Akteuren üblich sind, zentrale Akteure be-

reits Produktverantwortung übernommen haben und verschiedene Instrumente erfolgreich angewendet wurden.

- Für Holzmöbel steht eine IPP eher noch am Anfang. Hier gibt es nur wenig Kooperationen oder Kommunikation zwischen den Akteuren, diese haben zumeist nur einen Abschnitt des Lebensweges im Blick und die vorhandenen Instrumente zielen eher auf punktuelle Verbesserungen.

\section{Wahrgenommener IPP-Bedarf}

Durch die zunehmend länderübergreifende Ausdifferenzierung des jeweiligen Produktlebenswegs und insbesondere die Entsorgungsproblematik kündigt sich in beiden Produktbereichen ein Handlungsdruck zur akteursuibergreifenden Kooperation entlang des Produktlebenszyklus an. Insbesondere die Entsorgung von Kühlgeräten ist nicht allein über den Verordnungsweg regelbar. Sie erfordert ein System der freiwilligen, geteilten Produktverantwor-

\begin{tabular}{|c|c|c|c|c|}
\hline \multirow[t]{2}{*}{ Holzmöbel } & \multicolumn{4}{|c|}{ Maßnahmen in zentralen Problembereichen } \\
\hline & Forstwirtschaft & Emissionen & Nutzungsdauer & Entsorgung \\
\hline Staatliche & & - TA Luft und VOC-Richtlinie & Modellprojekte ZU & - TA Siedlungsabfall \\
\hline \multirow[t]{5}{*}{ Maßnahmen } & & für Emissionen in der Produktion & Möbelrücknahme und & Verordnung zur \\
\hline & & - $\mathrm{PCP}$ und Formaldehyd in der & Aufarbeitung & Holzentsorgung \\
\hline & & Chemikalienverbotsverordnung & & (in Vorbereitung) \\
\hline & & - Produktkennzeichen & & \\
\hline & & - Öffentliches Beschaffungswesen & & \\
\hline Maßnahmen & Produktkenn- & Forschung zu & "Flexible" Möbel & Recyclinggerecht \\
\hline anderer & zeichen/Siegel & wasserlöslichen Lacken & (mitwachsende & konstruierte Möbel \\
\hline \multirow[t]{2}{*}{ Akteure } & & - Verbraucherinformation & Kindermöbel, & \\
\hline & & & Regalsysteme etc.) & \\
\hline
\end{tabular}

Quelle: eigene Darstellung tung, das über die Entsorgungsproblematik hinaus Verbesserungen in der Produktentwicklung, der Herstellung, dem Vertrieb und der Nutzung des Produkts bewirkt. Im Fall der Holzmöbel beklagten viele Befragte eine Unübersichtlichkeit produktpolitischer Maßnahmen, deren Transparenz durch eine IPP verbessert werden könnte.

Zwar besteht in beiden Bereichen Interesse und Bedarf an einer Kooperation im Sinne einer IPP, andererseits ist unklar, welches ihre Themen und Ziele sein könnten. Insbesondere die praktischen Implikationen sind bisher noch wenig griffig. Es fehlt an praktischen Erfahrungen und einer Übersetzung des Mehrwerts einer IPP.

Es bietet sich an, die praktischen Implikationen von IPP im Rahmen von Pilotprojekten zu testen und zu erproben und damit die bisherige Abstraktheit des Themas zu verlassen. Wie aber sollten derartige Pilotprojekte beschaffen sein? Um welche Produkte sollte es sich handeln, welche Akteure sollten sich zusammensetzen und mit welchen Fragestellungen? Wer sollte das Pilotprojekt durchführen und zu welchen praktischen Ergebnissen kann es kommen?

\section{Produkte oder Bedürfnisfelder?}

Die Wahl des Ansatzes und der Prioritätensetzung des Pilotprojekts wird wesentlich darüber bestimmen, welche Akteure beteiligt werden und welche Art von Ergebnissen zu erwarten sind. Zu unterscheiden ist einerseits ein eher produktgruppenorientiertes Vorgehen, das dem dänischen Beispiel der ,product panels“ (2) folgen würde. Diese Panels bestehen aus den relevanten Akteuren, die an dem Lebensweg der Produktgruppen - in unserem Fall wären dies Kühlgeräte und Holzmöbel beteiligt sind. Sie sollen Aktions- und Maßnahmenpläne ausarbeiten, spezifische Ziele festlegen und deren Zielerreichung regelmäßig evaluieren. Bei dieser Prioritätensetzung ist eine Abgrenzung des Themas relativ einfach.

Ein anderer Ansatz ordnet die Produkte sogenannten Bedürfnisfeldern, hier Wohnen und Kühlen, zu und setzt an letzteren an. Dieser systemische Ansatz entspricht dem der Arbeiten der Enquete-Kommission „Schutz des Menschen und der Umwelt" und betrachtet nicht nur Produkte, sondern ihre Funktion für die KonsumentInnen. Ein solches Herangehen wird eher innovationsorientiert sein. Im Fall Kühlen zielte es nicht nur auf Produktverbesserungen bei Kühlgeräten ab, sondern auch auf systemische Innovationen wie Veränderungen im Wohnungsbau. Allerdings ist dieser systemische Ansatz in der Praxis schwerer zu handhaben. 


\section{Pilotprojekte: Initiatoren und Akteure ...}

In idealtypischer Hinsicht wird ein Pilotprojekt vom BMU bzw. Umweltbundesamt in Absprache mit den relevanten Akteuren entlang des Lebenswegs eines bestimmten Produkts angeregt. Es kann beispielsweise in Form von entsprechend vorbereiteten Workshops durchgeführt werden, unter Leitung eines/einer von allen Akteuren anerkannten erfahrenen MediatorIn. Das Projekt sammelt die produktbezogenen Erfahrungen und Einschätzungen der Akteure und fragt, wo ökologischer und politischer Handlungsbedarf gesehen wird, produktbezogene Problemlösungsansätze vorhanden sind und wo sich gemeinsame produktbezogene und ökologische Ziele abzeichnen.

Je nach gewähltem Fokus werden die relevanten Akteure zur Teilnahme aufgefordert. Dies kann Verbände betreffen und ökonomisch bedeutsame Schlüsselakteure, die eine große Gestaltungsmacht haben und verbandspolitische Aktivitäten stimulieren können (Verbandsmodell). Beim sogenannten Change-Agents-Modell werden hingegen zunächst maßgebliche ökologische Trendsetter und Innovatoren zu einer Mitarbeit eingeladen, um Innovationsbündnisse zu schaffen. Andererseits haben solche Teilnehmer eine geringe Gestaltungsmacht und - kurzfristig - keine große ökonomische Bedeutung, was für eine Öffnung zum Verbandsmodell spräche.

\section{... sowie Themen und Vorgehen}

Die Pilotprojekte setzen thematisch an den oben skizzierten ökologischen Herausforderungen der Produkte und an einer Bestandsaufnahme bisheriger Maßnahmen der verschiedenen Akteure entlang des Produktlebenswegs an. Sie werden deshalb durch ein sorgfältiges screening wissenschaftlicher Daten und Wissensbestände vorbereitet. Darauf aufbauend können von den Akteuren mögliche geteilte Ziele und Visionen für eine produktgruppenspezifische IPP entwickelt werden.

In jedem Fall werden Pilotprojekte am Handlungsbedarf aus Sicht dieser Akteure, ihren Bedürfnissen und bisherigen Erfahrungen ansetzen müssen. Bei den Kühlgeräten beispielsweise betrifft dies die Förderung des Marktzugangs für energieeffiziente Geräte und Innovationen, ferner akteursübergreifende Maßnahmen für eine qualitätsgesicherte Entsorgung, die Schaffung von Märkten für Recyclingprodukte und unter anderem auch eine Harmonisierung von DIN-Normen hinsichtlich ihrer Eignung zur Unterstïtzung des Ecodesigns der Produkte.
Bei den Holzmöbeln stellen die Akteure vor allem eine bessere Verbraucherinformation über verschiedene Produktkennzeichnungen, die Harmonisierung der Umweltzeichen und eine entsprechende Verkaufsberatung in den Vordergrund gemeinsamen Handlungsbedarfs. Ferner sollte die Weitergabe ökologisch relevanter Produktinformationen in den Vorketten verbessert und eine koordinierte Organisation der Möbelrücknahme erreicht werden. Unter Berücksichtigung des Lebenszyklus müssen aus ihrer Sicht auch Zerlegbarkeit, Trennbarkeit oder Reparaturfähigkeit der Möbel hinsichtlich der ökologischen Herausforderungen durch entsprechende Maßnahmen abgestimmt werden.

\section{Fazit}

Die beiden Fallbeispiele zeigen, dass die Ausgangsbedingungen der Branchen für eine IPP zwar sehr unterschiedlich sind, sich in beiden Fällen aber aufgrund ökologischer und ökonomischer Herausforderungen die Notwendigkeit zur akteursübergreifenden Kooperation im Sinne

\section{Weitere Literałur zum Schwerpunkł}

- Ecocycle Commission (1997): A Strategy for Sustainable Materials and Products.

Stockholm 1997.

Ernst \& Young/ SPRU: Developing the Foundation for Integrated Product Policy in the EU. London 2000. Kurzfassung unter http://europa.eu.int/ comm/environment/ipp/home.htm

- European Commission - DG XI: Workshop on Integrated Product Policy - 8 December 1998. Final report. Brüssel 1999. Download: http://europa.eu.int/ comm/environment/

ipp/home.htm

Nordic Council of Ministers: Proposal for a common Nordic IPP. Background documents prepared for a Nordic IPP-meeting in Saltsjöbaden February 9-10 in the year 2000. Kopenhagen 2000.

Oosterhuis, Frans/ Rubik, Frieder/ Scholl, Gerd: Product Policy in Europe. New Environmental Perspectives. Dordrecht et al. 1996.

Rubik, Frieder/ Teichert, Volker: Ökologische Produktpolitik - Von der Beseitigung von Stoffen und Materialien zur Rückgewinnung in Kreisläufen. Stuttgart 1997

VROM (Dutch Ministry of Housing, Physical Planning and Environment): Policy Document on Products and the Environment. Den Haag 1994. einer IPP abzeichnet. Der Zusatznutzen einer IPP bestünde jedoch nicht allein in der akteursübergreifenden Koordination von Maßnahmen, sondern in der Orientierung dieser Maßnahmen auf ein übergeordnetes ökologisches Ziel für diese Produktgruppe, an dem sich alle betreffenden Akteure orientieren.

Der Versuch, konkrete Schritte eines IPP-Pilotprojektes für die beiden Bereiche zu skizzieren, zeigt darüber hinaus, dass ein kooperativer Politikstil bzw. die Bereitschaft der Akteure zur Kooperation Voraussetzung und eigentlicher Motor einer IPP ist. Das bedeutet, dass IPP im glücklichsten Falle einen Prozess der verbindlichen ökonomischen und gesellschaftlichen Umgestaltung im Sinne der Agenda 21 einleitet. Es wäre aber ein Missverständnis, wollte man in ihr ein neues Instrument sehen, das im Sinne mechanistischer Steuerungsvorstellungen akteurs- und branchenübergreifend angewandt werden könnte.

\section{Anmerkungen}

(1) Rubik, Frieder/Simshäuser, Ulla/Hoffmann, Esther: Innovationen durch die Umweltpolitik - Integrierte Produktpolitik (IPP) in Deutschland. IÖW, Heidelberg/ Berlin 2000.

(2) Elektronikindustrien (Hrsg.): The Danish Electronics Product Area Panel. Action Plan 1999. A plan for production and use of more environmentally sound electronic products in Denmark, Kopenhagen 1999.

\section{Die Autorinnen}

Esther Hoffmann und Ulla Simshäuser sind wissenschaftliche Mitarbeiterinnen am Institut für ökologische Wirtschaftsforschung.

Kontakt: IÖW-Regionalbüro Baden-Württemberg, Bergstraße 7, 69120 Heidelberg, Tel. 06221/ 64916-0, Fax 06221/ 27060, E-mail: mailbox@heidelberg.ioew.de (Ulla Simshäuser), esther.hoffmann@ioew.de

\section{Sie wollen eine Anzeige schalten?}

Bei Fragen rund um Anzeigen steht Ihnen unsere Anzeigenvertretung gerne zur Verfügung.

Kontakt:

BN-Service Agentur, Jochen Pfeiffer

Fon ++49/(0)9123/94 4919

E-mail

anzeigen@service.bund-naturschutz.de 
(c) 20I0 Authors; licensee IÖW and oekom verlag. This is an article distributed under the terms of the Creative Commons Attribution Non-Commercial No Derivates License (http://creativecommons.org/licenses/by-nc-nd/3.o/), which permits unrestricted use, distribution, and reproduction in any medium, provided the original work is properly cited. 